\title{
ESTUDO DO PROCESSO DE TORREFAÇÃO DE RESÍDUOS DE BIOMASSAS PARA FINS ENERGÉTICOS
}

STUDY OF THE TORREFACTION PROCESS BIOMASS WASTES FOR ENERGY PURPOSES

\section{Allison Gonçalves Silva}

Químico pela Universidade Estadual de Santa Cruz (UESC) - Ilhéus (BA). Doutor em Química pela Universidade Federal da Bahia (UFBA) -Salvador (BA). Docente do Instituto Federal de Educação, Ciência e Tecnologia da Bahia (IFBA) - Porto Seguro (BA), Brasil.

\section{Thaise Alves dos Santos}

Química pelo IFBA. Pós-graduanda em Ciência e Tecnologia Ambiental. Docente do IFBA - Porto Seguro (BA), Brasil.

\section{Marcus Luciano Silva de Ferreira Bandeira}

Químico pela UFBA. Doutor em Química pela UFBA-Salvador (BA). Docente do IFBA - Porto Seguro (BA), Brasil.

\section{Priscila Ferreira de Oliveira}

Química pela Universidade Federal Rural do Rio de Janeiro (UFRRJ). Doutora em Ciência e Tecnologia de Polímeros pela Universidade Federal do Rio de Janeiro (UFRJ) Rio de Janeiro (RJ). Docente do Instituto Federal de Educação, Ciência e Tecnologia Baiano (IFBaiano) - Teixeira de Freitas (BA), Brasil.

\section{Endereço para correspondência:}

Allison Gonçalves Silva Rodovia BR-367, km 57,5, s/n Fontana I - 45810-000 - Porto Seguro (BA), Brasil E-mail: allisongoncalves@ifba.edu.br

Recebido: 26/01/2017

Aceito: 30/06/2017

\section{RESUMO}

O objetivo deste trabalho foi estudar resíduos de coco, cacau e dendê culturas largamente encontradas no Extremo Sul da Bahia -, verificando o efeito da torrefação a diferentes temperaturas $\left(230,240\right.$ e $\left.250^{\circ} \mathrm{C}\right)$ sobre essas biomassas, de modo a observar sua influência no poder calorífico superior (PCS) por meio de análise imediata, termogravimétrica e de microscopia eletrônica de varredura. A caracterização físico-química dos materiais secos e torrificados apontou uma tendência à diminuição dos teores de voláteis e de umidade, aumento dos teores de carbono fixo, de cinzas e do PCS, sendo essas características mais acentuadas a temperaturas mais altas. De modo geral, os experimentos realizados contribuíram para o maior potencial energético dos resíduos estudados. A biomassa de coco apresentou o maior PCS, sendo 24,59 MJ.kg-1. Para a biomassa de coco torrificada, o aumento médio no PCS, com relação à biomassa seca, foi de $24,1 \%$, para cacau $21,3 \%$ e para dendê $35,2 \%$.

Palavras-chave: Extremo Sul da Bahia; biocombustíveis; dendê; cacau; coco.

\section{ABSTRACT}

The purpose of this work was to study coconut, cocoa and palm oil biomass products, all which are widely found in the Extreme South of Bahia, to investigate the effect of torrefaction at various temperatures $(230,240$ and $250{ }^{\circ} \mathrm{C}$ ) of these biomasses, and to verify the influence of torrefaction on the higher heating value (HHV), through proximate analysis, thermogravimetric and scanning electron microscopy. Results from the physical and chemical characterization of the dry torrefied materials indicated a trend of lower volatiles and moisture and higher fixed carbon content, ash content, and $\mathrm{HHV}$, especially when subjected to the highest temperatures. In general, the experiments resulted in a higher energy potential for each biomass that was studied. The coconut biomass had the largest observed $\mathrm{HHV}$ at $24.59 \mathrm{MJ}^{\mathrm{kg}} \mathrm{kg}^{-1}$. Across all experiments, the dry torrefied coconut, cocoa and palm oil had average percent increases in HHV of $24.1 \%, 21.3 \%$ and $35.2 \%$, respectively.

Keywords: Extreme South of Bahia; biofuels; palm oil; cocoa; coconut. 


\section{INTRODUÇÃO}

Atualmente, observam-se várias pesquisas que visam ao desenvolvimento de diferentes fontes energéticas alternativas, para atender à demanda de energia mundial e contribuir para a redução do consumo de combustíveis fósseis e da agressão ao meio ambiente, evidenciando os conceitos de bioeconomia e sustentabilidade para o uso de biomassa (MUIZNIECE et al., 2016; NAM et al., 2016; AGAR et al., 2015; AZADI et al., 2017).

No Brasil, a utilização de biocombustíveis gerados a partir de biomassa está entre as principais alternativas energéticas, tornando-se instrumento de extrema importância para redução da emissão de gases de efeito estufa na atmosfera; além disso, a cogeração de energia elétrica por meio de resíduos de biomassa também é uma fonte energética de grande potencial. $O$ desenvolvimento de políticas públicas que incentivam o uso de biocombustíveis tende a crescer em razão da insegurança energética em âmbito mundial e do aumento dos preços de produtos relacionados diretamente a esse mercado. O Brasil possui perspectivas muito interessantes nesse sentido, sendo que as fontes renováveis têm uma participação de cerca de $50 \%$ em sua matriz energética, enquanto a média mundial é de aproximadamente $14 \%$. Com isso, é possível que o Brasil ganhe grande visibilidade internacional no mercado bioenergético, o que torna este um setor economicamente promissor para o país (CNI, 2007; LOBÃO, 2008; SILVA et al., 2005).

A biomassa energética apresenta rotas significativamente diversificadas, com extensa variedade de fontes, que vão desde os resíduos agrícolas, industriais e urbanos até as culturas dedicadas, com grande quantidade de tecnologias para os processos de conversão, tais como pirólise, gaseificação e combustão. Tais tecnologias têm sido cada vez mais aprimoradas, de modo a explorar ao máximo a eficiência da biomassa como alternativa energética (DODDAPANENI et al., 2016). Diferentes procedimentos têm sido investidos a fim de melhorar as propriedades físicas da biomassa sólida, dentre os quais os tratamentos mecânicos, tais como a peletização e a briquetagem, que resultam em um produto da biomassa homogêneo e de alta densidade energética (CHAO et al., 2015; IBRAHIM et al., 2013; CHEN et al., 2017; RUDOLFSSON et al., 2017). Outro tratamento aplicado para o condicionamento da biomassa é a torrefação. Este utiliza temperaturas mais baixas do que a pirólise e a gaseificação, resultando em um sólido com caracte- rísticas energéticas superiores às da biomassa in natura. Quando a torrefação é associada à peletização, um biocombustível com alto potencial energético é obtido e pode ser usado na própria indústria em caldeiras e geradores de energia (SYAMSIRO et al., 2012; STELTE, 2013; BRASIL, 2007; SILVA et al., 2005).

A torrefação da biomassa desenvolve-se entre 200 e $300{ }^{\circ} \mathrm{C}$, embora alguns autores recomendem não ultrapassar o limite de $280^{\circ} \mathrm{C}$, sendo que, nessas condições, a umidade é removida e a hemicelulose é degradada, provocando a liberação de ácido acético, frações de fenol e outros compostos de baixo poder calorífico (CIOLKOSZ; WALLACE, 2011; STELT et al., 2013; PRINS et al., 2006; CORTEZ et al., 2008).

Em trabalho realizado por Chen et al. (2016), foi estudado o efeito do processo de torrefação a temperaturas de 225 a $300{ }^{\circ} \mathrm{C}$ em resíduos de pinheiro. Os autores concluíram que, quando a biomassa é torrificada a $250{ }^{\circ} \mathrm{C}$, grande parte do bio-óleo produzido conduz a um menor teor de ácidos orgânicos, bem como a uma diminuição do teor de cinzas, demonstrando a importância do processo de torrefação antes de a biomassa ser pirolisada.

A utilização de resíduos de trigo e de arroz como biomassa para geração de energia foi proposta por Ciolkosz e Wallace (2011), que verificaram que as reações que ocorrem numa temperatura entre 200 e $300{ }^{\circ} \mathrm{C}$ são essencialmente: desvolatilização e carbonização da hemicelulose; despolimerização e desvolatilização de uma pequena parte da lignina (amolecimento); despolimerização e desvolatilização de parte da celulose. Como resultado, tem-se um rendimento de $70 \%$ de massa, rendimento energético de $90 \%$, poder calorífico $17-19 \mathrm{MJ} \mathrm{kg}^{-1}$ (poder calorífico inferior) para $18-23 \mathrm{MJ} \mathrm{kg}^{-1}$ e com absorção de umidade entre 1 e $6 \%$.

A utilização promissora da biomassa se dá também por diversos segmentos industriais e agroindustriais que precisam ser reaproveitados ou descartados corretamente para agregarem valor a variados processos produtivos (SABIL et al., 2013; ARIAS et al., 2008; CHEN; KUO, 2010; PROSKURINA et al., 2017; LASEK et al., 2017).

Nam et al. (2016) realizaram o processo de torrefação de três tipos de resíduos de biomassa, incluindo cachos de frutas, aglomerados de madeira e cascas de arroz, sendo avaliados em escala piloto, observando que esses resíduos 
apresentam uma quantidade de absorção de água muito grande, o que diminui substancialmente o poder calorífico, sendo necessário o condicionamento dessas biomassas.

As biomassas selecionadas para serem estudadas no presente trabalho foram a casca de coco, a casca de cacau e a fibra de dendê, coletadas nos municípios de Porto Seguro e de Belmonte. De acordo com estimativas divulgadas pela Superintendência de Estudos Econômicos e Sociais da Bahia (SEl, 2015), no ano de 2013 o Extremo Sul da Bahia contou com um total de 46.877 hectares de área plantada e colhida da cultura de cacau, 11.030 hectares de área plantada e colhida de coco e 9 hectares de área plantada e colhida de dendê - o que equivale a 15.288 toneladas de cacau, 70.117.000 frutos de coco e 42 toneladas de dendê. A exemplo de resíduos, segundo a Empresa Brasileira de Pesquisa Agropecuária (Embrapa), o Brasil produz cerca de dois bilhões de cocos por ano e cada fruto gera aproximadamente $1 \mathrm{~kg}$ de resíduo sólido, o que corresponde a cerca de $80 \%$ do peso bruto (SALES, 2011; A LAVOURA, 2012; MORORÓ, 2007; CEPLAC, 2014; EMBRAPA, 2012; CONAB, 2006; SEI, 2015).

Nesse contexto, a utilização dessas biomassas para a produção de biocombustíveis sólidos, por meio da torrefação, constitui uma importante alternativa para agregar valor produtivo a essas culturas e para auxiliar no avanço do desenvolvimento sustentável do Extremo Sul da Bahia. Dessa forma, o presente trabalho teve como objetivo estudar resíduos de coco, cacau e dendê, culturas largamente encontradas no Extremo Sul da Bahia, verificando o efeito da torrefação a diferentes temperaturas $-230,240$ e $250^{\circ} \mathrm{C}$ - sobre essas biomassas, observando, assim, sua influência no poder calorífico por meio de análise imediata, termogravimétrica e de microscopia eletrônica de varredura.

\section{MATERIAIS E MÉTODOS}

\section{Pré-tratamento dos resíduos}

Realizou-se o pré-tratamento das biomassas conforme o Standard Test Method for Moisture Analysis of Particulate Wood Fuels (ASTM) E 871-82 (ASTM, 1998a). Em seguida, triturou-se as biomassas (coco, dendê e cacau)

\section{Processo de torrefação}

Realizou-se a torrefação em forno mufla em que era alimentado com gás de arraste, inerte hélio $(\mathrm{He})$. As temperaturas de torrefação utilizadas foram 230, 240 e $250^{\circ} \mathrm{C}$. Todos os experimentos de torrefação foram realizados utilizando $5 \mathrm{~g}$ dos resíduos de biomassas em base seca e peneiradas (diâmetro de partícula entre 80 e 100 mesh), sendo aquecidos a uma

\section{Análise imediata da biomassa}

Inicialmente todas as amostras foram secas conforme ASTM E 871-82, que se refere ao teor de umidade. Em seguida, os materiais foram caracterizados por análise imediata: o teor de umidade foi obtido pela ASTM E 871-82; o teor de materiais voláteis foi de-

\section{Estimativa do poder calorífico superior}

A fim de avaliar o efeito da temperatura de torrefação, foram determinados os poderes caloríficos superiores em um moinho de facas para posterior realização de todos os procedimentos e análises deste estudo (ASTM E $871-82,1998$ ). Esse método visou a diminuir o tamanho das partículas e a facilitar o processo de torrefação.

taxa de $10^{\circ} \mathrm{C} \cdot \mathrm{min}^{-1}$ até a temperatura de torrefação e mantida por tempo programado, que foi de $60 \mathrm{~min}$. Os resíduos torrificados foram armazenados em frascos para posterior caracterização (SILVA, 2013). O processo de torrefação visa a eliminar a hemicelulose, componente responsável pela maior absorção de umidade.

terminado pela ASTM E 872-82; o teor de cinzas foi determinado pela ASTM D 1102-84; e o teor de carbono fixo foi obtido por meio de uma relação entre os teores de materiais voláteis e de cinzas (EMBRAPA, 2012; ASTM, 1995; 1998b).

(PCS) para os resíduos em estudo, em MJ.kg-1 de acordo com a Equação 1. Essa equação é uma correlação 
estabelecida por Parikh et al. (2005) em seus estudos, por meio da qual é possível fazer estimativas do PCS de materiais, que variam desde materiais carbonáceos sólidos até todos os tipos de material de biomassa e carvão combustível derivados de resíduos, por meio de sua análise imediata (PARIKH et al., 2005).

\section{Análise termogravimétrica}

Com a finalidade de quantificar os teores dos principais macrocomponentes presentes nas biomassas - celulose, hemicelulose e lignina -, realizou-se a análise termogravimétrica em um equipamento da empresa (marca) TA Instruments (modelo Q 500).

\section{Microscopia eletrônica de varredura}

A técnica de microscopia eletrônica de varredura (MEV) foi empregada a fim de verificar a morfologia das superfícies das fibras tratadas, pelas quais se pode avaliar se houve a remoção, ainda que parcial, dos extraíveis amorfos das fibras. Para isso, as amostras foram fixadas em porta amostra com fita adesiva de carbono e metalizou-se com vapor de ouro por 1 minuto e 30

$$
\mathrm{PCS}=0,3536 \mathrm{CF}+0,1559 \mathrm{MV}-0,0078 \mathrm{ASH}
$$

Em que CF é o teor de carbono fixo; MV, o teor de voláteis; e ASH, o teor de cinzas - todos em percentual, obtidos nas análises das amostras.

Foram utilizados cerca de $10 \mathrm{mg}$ da amostra; empregou-se uma taxa de aquecimento de $10^{\circ} \mathrm{C} \cdot \mathrm{min}^{-1}$, variando da temperatura ambiente até $700^{\circ} \mathrm{C}$ sob atmosfera inerte de nitrogênio $\left(\mathrm{N}_{2}\right)$ com fluxo de $50 \mathrm{~mL} \cdot \mathrm{min}^{-1}$.

segundos em metalizador da marca Quorum (modelo Q150R) para condução de elétrons e também para evitar o acúmulo de cargas com consequente destruição das amostras. As análises foram conduzidas no equipamento com detector de elétrons secundário, em MEVTescan (modelo Vega 3 LMU).

\section{RESULTADOS E DISCUSSÃO}

Os resultados encontrados por meio das análises das biomassas de coco, cacau e dendê, secas e torrificadas, possibilitaram uma avaliação acerca dos principais efeitos da torrefação nas propriedades físico-químicas dos materiais estudados.

O aspecto característico da biomassa torrificada pode ser observado na Figura 1, na qual se verifica o escurecimento gradual com o aumento da temperatura de torrefação.

Na Tabela 1, são apresentadas as principais características dos resíduos de biomassas, no que se refere à caracterização por análise imediata, bem como aos valores de PCS.

De acordo com o estudo de Chai e Saffron (2016), a presença de umidade na biomassa é importante em níveis adequados, pois processos mecânicos como a peletização dependem da quantidade adequada de água presente na matriz da biomassa a ser processada, de modo a aumentar a durabilidade dos péletes produzidos. No entanto, altos teores de umidade acarream em menor potencial energético, pois a presença de água contribui para a inserção de energia que será consu- mida em sua vaporização, para que depois se inicie a decomposição da biomassa (WANG et al., 2017a).

Observa-se nos resultados expostos na Tabela 1 que há uma tendência ao aumento do poder calorífico devido à diminuição do teor de umidade nas biomassas torrificadas. Para a biomassa de coco seca, o PCS encontrado foi de $19,30 \mathrm{MJ}^{\mathrm{kg}}{ }^{-1}$, com um aumento de $22,71 \mathrm{MJ} . \mathrm{kg}^{-1}$ para os resíduos torrificados a $230^{\circ} \mathrm{C}, 22,57{\mathrm{MJ} . \mathrm{kg}^{-1} \text { para os }}^{\circ}$ torrificados a $240^{\circ} \mathrm{C}$ e $22,59 \mathrm{MJ}^{\mathrm{kg}} \mathrm{kg}^{-1}$ para os torrificados a $250^{\circ} \mathrm{C}$. Para os resíduos de cacau in natura, o PCS estimado foi de 19,12 MJ.kg-1 aumentando para 22,54 MJ.kg-1

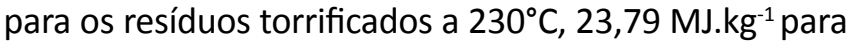
os torrificados a $240^{\circ} \mathrm{C}$ e $23,24 \mathrm{MJ}^{\circ} \mathrm{kg}^{-1}$ para os torrificados a $250^{\circ} \mathrm{C}$. E, para a fibra de dendê, o PCS foi de 17,05 $\mathrm{MJ} . \mathrm{kg}^{-1}$, subindo para $22,70 \mathrm{MJ}^{\mathrm{kg}}{ }^{-1}$ para os resíduos tor-

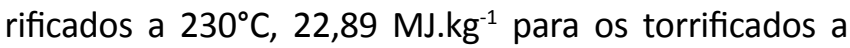
$240^{\circ} \mathrm{C}$ e $23,54 \mathrm{MJ}^{-\mathrm{kg}^{-1}}$ para os torrificados a $250^{\circ} \mathrm{C}$. Essas observações estão de acordo com a literatura, indicando que grande parte do fenômeno em questão se deve à degradação do componente menos estável termicamente presente em biomassas, a hemicelulose, conforme estudos realizados por Wang et al. (2017b), que ava- 
liaram o efeito da torrefação sobre as características de degradação da hemicelulose e seus subprodutos quando submetidos a pirólise.

Para a biomassa de coco, foi possível verificar que, de acordo com o aumento da temperatura de torrefação, houve a diminuição dos teores de umidade, partindo de $10,22 \%$ na biomassa seca para $4,98 \%$ na biomassa torrificada a $230^{\circ} \mathrm{C}, 3,29 \%$ na torrificada a $240^{\circ} \mathrm{C}$ e $3,20 \%$ na torrificada a $250^{\circ} \mathrm{C}$; e de voláteis, $78,88 \%$ na biomassa seca caindo para $58,83 \%$ na torrificada a $230^{\circ} \mathrm{C}, 46,85 \%$ na torrificada a $240^{\circ} \mathrm{C}$ e $46,17 \%$ na torrificada a $250^{\circ} \mathrm{C}$. Observou-se também o aumento dos teores de carbono fixo, indo de 19,84 para $38,4,48,92$ e $49,28 \%$ nas biomassas secas e torrificadas a 230,240 e $250^{\circ} \mathrm{C}$, respectivamente; e também do teor de cinzas aumentando de
1,28 para $2,82,4,23$ e $4,56 \%$ nas biomassas secas e torrificadas a 230,240 e $250{ }^{\circ} \mathrm{C}$, respectivamente.

O teor de cinzas da biomassa de coco seca foi inferior aos valores encontrados por Nakashima et al. (2014) para as biomassas in natura de bagaço de cana-de-açúcar, palha de milho, capim elefante e podas de jardim, o que é interessante em razão do maior teor de cinzas contribuir negativamente durante os processos de combustão (NANDA et al., 2016; BERGMAN; KIEL, 2005; VIEIRA, 2012).

O comportamento observado na análise imediata das biomassas de cacau foi bastante semelhante ao observado para as fibras de coco. Os teores de voláteis e de umidade diminuíram, o que contribuiu para o aumento do PCS; para voláteis, os valores variaram de $71,14 \%$ nos re-

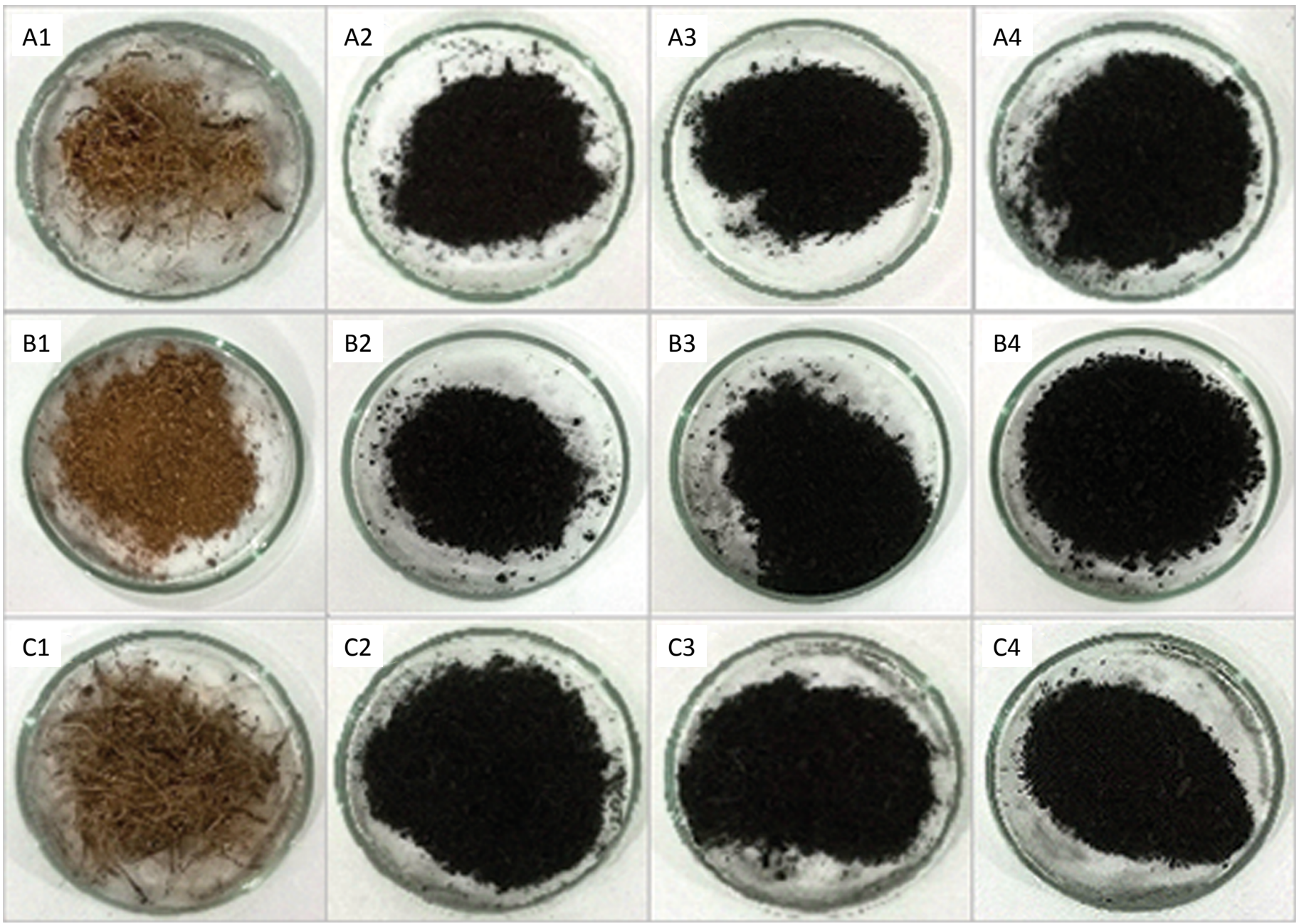

Figura 1 - Biomassa de coco: $\mathrm{A} 1$, in natura; de $\mathrm{A} 2$ a $\mathrm{A} 4$, torrificadas a 230,240 e $250^{\circ} \mathrm{C}$, respectivamente. Biomassa de cacau: $\mathrm{B} 1$, in natura; de $\mathrm{B} 2$ a B4, torrificadas a 230,240 e $250^{\circ} \mathrm{C}$, respectivamente. Biomassa de dendê: C1, in natura; de C2 a C4, torrificadas a 230,240 e $250^{\circ} \mathrm{C}$, respectivamente. 
síduos in natura para 47,59, 41,04 e 40,32\% nas biomassas torrificadas a 230,240 e $250{ }^{\circ} \mathrm{C}$, respectivamente. Os teores de umidade diminuíram consideravelmente, variando de 9,36\% nos resíduos secos para 1,46, 1,41 e $1,16 \%$ nas biomassas torrificadas a 230,240 e $250^{\circ} \mathrm{C}$, respectivamente. $\mathrm{O}$ teor de carbono fixo aumentou de $22,84 \%$ na biomassa seca para $42,96,49,38$ e $48,20 \%$ nos resíduos torrificados a 230,240 e $250^{\circ} \mathrm{C}$, respectivamente. $\mathrm{E}$ o teor de cinzas variou de $6,02 \%$ na biomassa in natura para 9,44, 9,58 e 11,48\% nos resíduos torrificados a 230,240 e $250^{\circ} \mathrm{C}$, respectivamente (NANDA et al., 2016; BERGMAN; KIEL, 2005; VIEIRA, 2012).

Para os resíduos de dendê foi observada a diminuição do teor de umidade, variando este de $9,42 \%$ na biomassa seca para 5,28, 3,91 e 3,63\% nos resíduos torrificados a 230,240 e $250{ }^{\circ} \mathrm{C}$, respectivamente. $\mathrm{O}$ teor de voláteis também diminuiu, indo de $77,53 \%$ na biomassa in natura para $53,89,53,17$ e $50,18 \%$ na biomassa torrificada a 230,240 e $250^{\circ} \mathrm{C}$, respectivamente. $\mathrm{O}$ teor de carbono fixo aumentou de $14,22 \%$ nos resíduos secos para $40,57,41,43$ e $44,55 \%$ nos resíduos torrificados a 230 , 240 e $250^{\circ} \mathrm{C}$, respectivamente. A diminuição dos teores de voláteis e de umidade e o maior teor de carbono fixo de acordo com o aumento da temperatura de torrefa- ção seguiram as mesmas tendências observadas para as biomassas de coco e de cacau; já o teor de cinzas seguiu tendência contrária, diminuindo conforme o aumento da temperatura. Os valores obtidos foram $8,24 \%$ para a biomassa seca, 5,54, 5,41 e 5,26\% para as biomassas torrificadas a 230,240 e $250{ }^{\circ} \mathrm{C}$, respectivamente.

A variação do teor de cinzas não segue uma tendência específica; é um fator que depende principalmente do material tratado, podendo ser influenciado pelo tratamento da cultura. Quanto mais alto o teor de cinzas, maiores as chances de se causar danos aos equipamentos de conversão térmica por meio da elevação do índice de slagging (incrustações). Desse modo, a diminuição do teor de cinzas para a biomassa de dendê é um fator muito importante, pois quando presentes no combustível as cinzas não são queimadas durante a combustão - geralmente, com composição química, silício $(\mathrm{Si})$, potássio $(\mathrm{K})$, sódio $(\mathrm{Na})$, enxofre $(S)$, cálcio $(\mathrm{Ca})$, fósforo $(\mathrm{P})$, magnésio $(\mathrm{Mg})$ e ferro (Fe) (CONAB, 2006; TAVARES \& SANTOS, 2013).

Segundo Tavares e Santos (2013), o poder calorífico superior está entre as características mais importantes para avaliar se há viabilidade na utilização de determinada biomassa vegetal na geração de energia. A esti-

Tabela 1 - Análise imediata e estimativa do poder calorífico superior dos materiais secos e torrificados (análises realizadas em triplicata).

\begin{tabular}{|c|c|c|c|c|c|}
\hline Biomassa & Análise elementar & Biomassa seca & $\begin{array}{c}\text { Torrificada } \\
230^{\circ} \mathrm{C}\end{array}$ & $\begin{array}{c}\text { Torrificada } \\
240^{\circ} \mathrm{C}\end{array}$ & $\begin{array}{c}\text { Torrificada } \\
250^{\circ} \mathrm{C}\end{array}$ \\
\hline \multirow{5}{*}{ Coco } & Voláteis (\%) & 78,88 & 58,83 & 46,85 & 46,17 \\
\hline & Cinzas (\%) & 1,28 & 2,82 & 4,23 & 4,56 \\
\hline & Carbono fixo (\%) & 19,84 & 38,34 & 48,92 & 49,28 \\
\hline & Umidade (\%) & 10,22 & 4,98 & 3,29 & 3,20 \\
\hline & PCS (MJ.kg-1) & 19,30 & 22,71 & 24,57 & 24,59 \\
\hline \multirow{5}{*}{ Cacau } & Voláteis (\%) & 71,14 & 47,59 & 41,04 & 40,32 \\
\hline & Cinzas (\%) & 6,02 & 9,44 & 9,58 & 11,48 \\
\hline & Carbono fixo (\%) & 22,84 & 42,96 & 49,38 & 48,20 \\
\hline & Umidade (\%) & 9,36 & 1,46 & 1,41 & 1,16 \\
\hline & PCS (MJ.kg-1) & 19,12 & 22,54 & 23,79 & 23,24 \\
\hline \multirow{5}{*}{ Dendê } & Voláteis (\%) & 77,53 & 53,89 & 53,17 & 50,18 \\
\hline & Cinzas (\%) & 8,24 & 5,54 & 5,41 & 5,26 \\
\hline & Carbono fixo (\%) & 14,22 & 40,57 & 41,43 & 44,55 \\
\hline & Umidade (\%) & 9,42 & 5,28 & 3,91 & 3,63 \\
\hline & PCS (MJ.kg-1) & 17,05 & 22,70 & 22,89 & 23,54 \\
\hline
\end{tabular}


mativa do PCS das biomassas em estudo foi feita com base nos teores de materiais voláteis, de carbono fixo e de cinzas (BERGMAN; KIEL, 2005).

Como se pode observar na Tabela 1, após a torrefação a tendência que predomina é um aumento do PCS dos biocombustíveis sólidos. Uma vez que o PCS sofre grande influência do teor de umidade, em razão da quantidade de energia que precisará ser disponibilizada para a vaporização da água contida no combustível, o fato de se observar a diminuição do conteúdo de umidade em todas as amostras torrificadas indica grande favorecimento do conteúdo energético desses resíduos após o condicionamento térmico (BERGMAN; KIEL, 2005).

Esses valores também permitem afirmar que, para as biomassas de coco e de dendê, a temperatura de torrefação que mais contribuiu para o aumento do PCS foi $250^{\circ} \mathrm{C}$, e para o cacau, a de $240^{\circ} \mathrm{C}$. Dentre as três biomassas analisadas, a que teve maior potencial energético, em todas as temperaturas de torrefação e in natura, foi a biomassa de coco. Isso pode estar relacionado com o teor de lignina presente nessa biomassa, pois, de acordo com Santos et al. (2016), a lignina possui porcentagens consideráveis de carbono elementar em sua composição, que irá gerar maiores teores de carbono fixo. A diminuição do PCS da biomassa de cacau torrificada a $250^{\circ} \mathrm{C}$, com relação às tendências dos demais materiais, também se dá em razão da relação entre os teores de cinzas, de voláteis e de carbono fixo.

Neste trabalho, assim como reportado na literatura, é possível verificar que as biomassas analisadas possuem PCS relativamente altos. Nakashima et al. (2014) encontraram valores de PCS de 17,54 MJ.kg-1 , 16,78 MJ.kg-1, 17,18 MJ.kg-1 e $16,63{\mathrm{MJ} . \mathrm{kg}^{-1}}$ para as biomassas in natura de bagaço de cana-de-açúcar, palha de milho, capim elefante e podas de jardim, respectivamente. Arias et al. (2008) verificaram o valor de $21,8 \mathrm{MJ}^{\mathrm{kg}}{ }^{-1}$ para o PCS da biomassa de eucalipto torrificada a $240^{\circ} \mathrm{C}$ por tempo de residência de $1 \mathrm{~h}$. Esse valor é mais baixo que o das biomassas de casca de coco, casca de cacau e fibra de dendê apresentados na Tabela 1.

Yue et al. (2017) investigaram o efeito do processo de torrefação nas temperaturas de 250,275 e $300^{\circ} \mathrm{C}$ de resíduos de sorgo, sendo observado aumento do poder

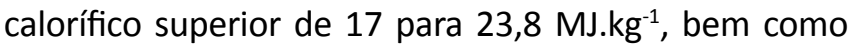
sua densidade energética. Brostrom et al. (2012) encon-

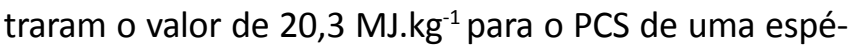
cie de madeira não tratada e valores entre 20,6 e 27,8
MJ.kg-1 para a mesma madeira torrificada a temperaturas acima de $500^{\circ} \mathrm{C}$ por tempo de residência entre 8 e 25 min. Apesar de os autores terem utilizado menor tempo de residência, as temperaturas de torrefação adotadas são bastante altas, fazendo com que alguns estudiosos classifiquem o processo térmico adotado por eles como pirólise, e não como torrefação. Almeida et al. (2010) verificaram valores de PCS entre 17,6 e 22,6 MJ.kg-1 para a biomassa de casca de eucalipto das espécies Eucalyptusgrandis e Eucalyptussaligna, torrificadas a temperaturas entre 220 e $280^{\circ} \mathrm{C}$ por tempo de residência de $1 \mathrm{~h}$, e valores entre 20,8 e $23,0 \mathrm{MJ} \mathrm{kg}^{-1}$ para a madeira da mesma biomassa torrificada às mesmas temperaturas e durante o mesmo tempo de residência, valores próximos aos encontrados neste trabalho. Chen e Kuo (2010) relacionaram os valores dos PCS das biomassas de bambu, salgueiro, casca de coco e figueira-benjamim in natura como 17,32, 18,37, 17,66 e 16,39 MJ.kg-1, respectivamente, sendo que os valores encontrados em nosso trabalho para as biomassas de cacau e de dendê in natu$r a$ apresentaram PCS maiores e a de coco um valor um pouco menor, porém ficando próximo ao valor encontrado pelos autores. Deng et al. (2009) investigaram o PCS da palha de arroz e do talo de colza, para a temperatura

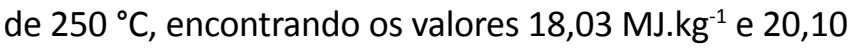
$\mathrm{MJ} . \mathrm{kg}^{-1}$, respectivamente. Buratti et al. (2017) encontra-

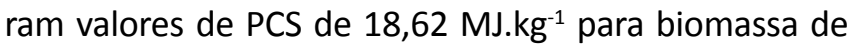
casca de café e 21,69 MJ. $\mathrm{kg}^{-1}$ para resíduo de café usado, sendo esses valores menores que os encontrados para as biomassas torrificadas a $250{ }^{\circ} \mathrm{C}$ no presente trabalho.

A diminuição do teor de umidade observada se deve à degradação do macrocomponente mais instável termicamente, hemicelulose, que possui uma temperatura de degradação baixa, em torno de 160 e $200^{\circ} \mathrm{C}$, e cuja presença indica que a biomassa possui grupos hidroxilas, o que favorece forte interação com moléculas de água por meio de ligações de hidrogênio, o que aumenta o teor de umidade, diminuindo o PCS. Isso pode ser confirmado pela análise termogravimétrica, cujos resultados são apresentados na Figura 2.

A degradação da hemicelulose inicia-se a cerca de $160{ }^{\circ} \mathrm{C}$; já a celulose e a lignina se decompõem a temperaturas mais elevadas, pois são mais resistentes à degradação e à decomposição térmica. A maioria das amostras torrificadas não apresentou o pico característico da hemicelulose. Tal fato indica que esse componente já não está presente nas biomassas após a tor- 
A

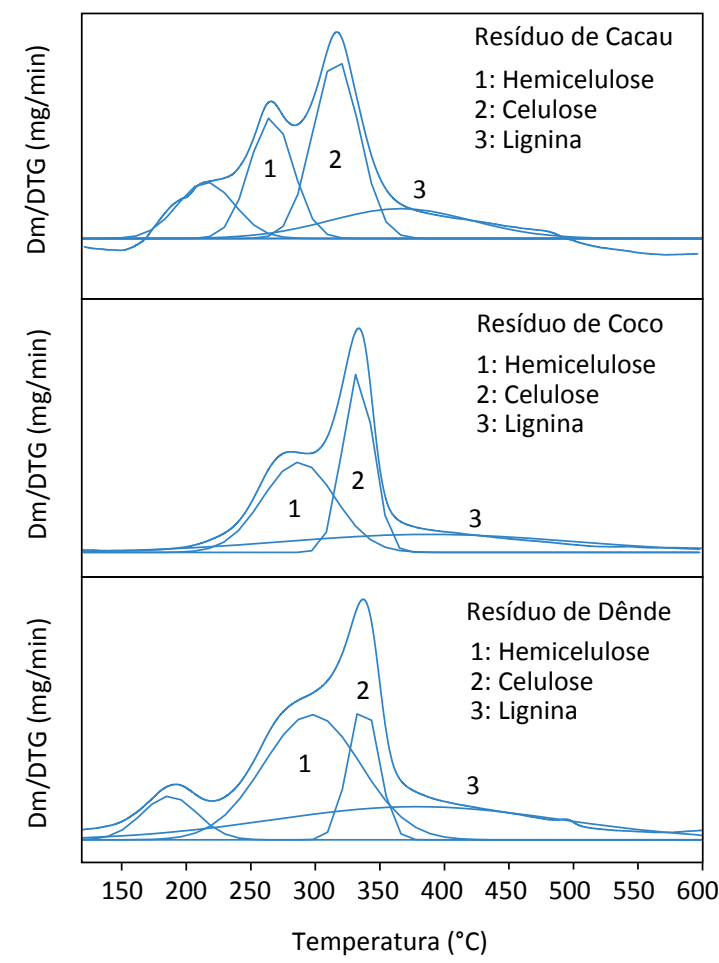

C

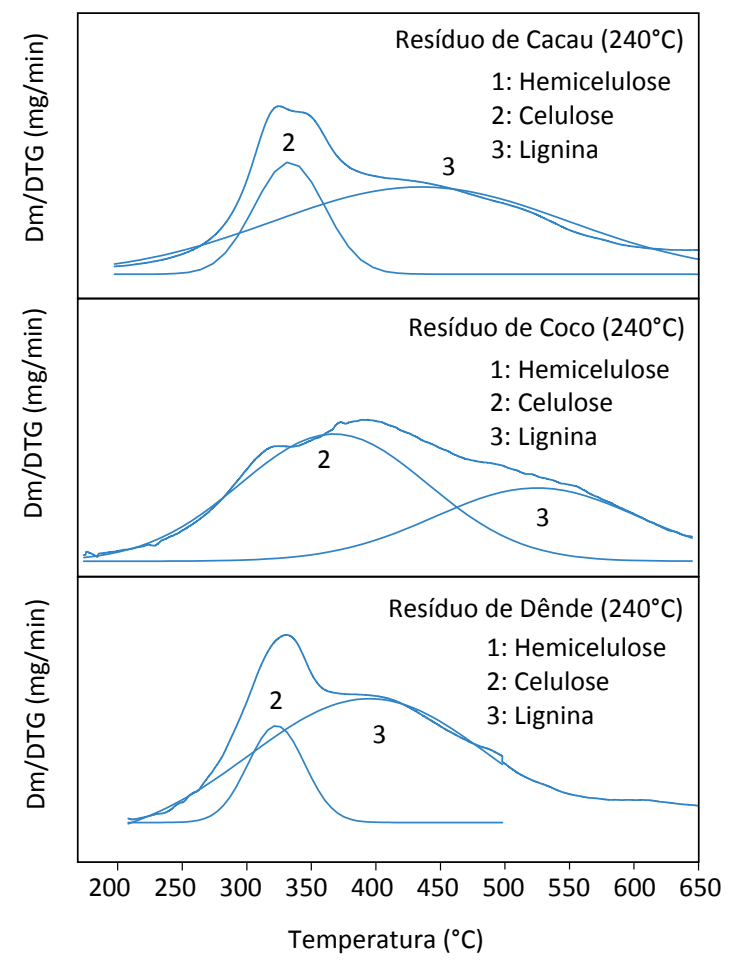

B

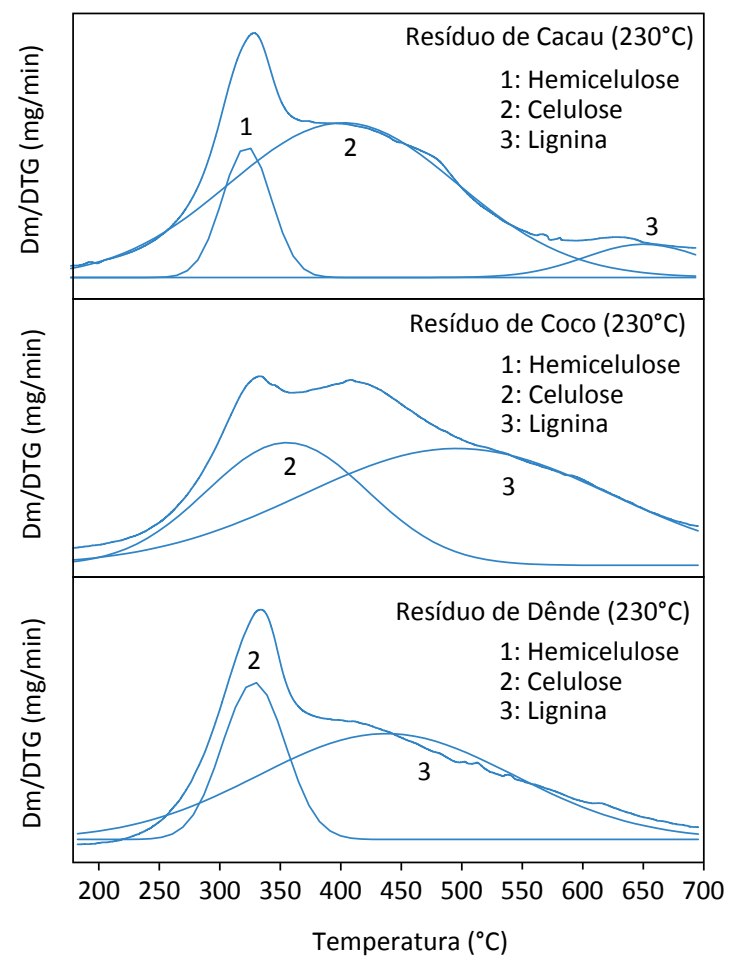

D

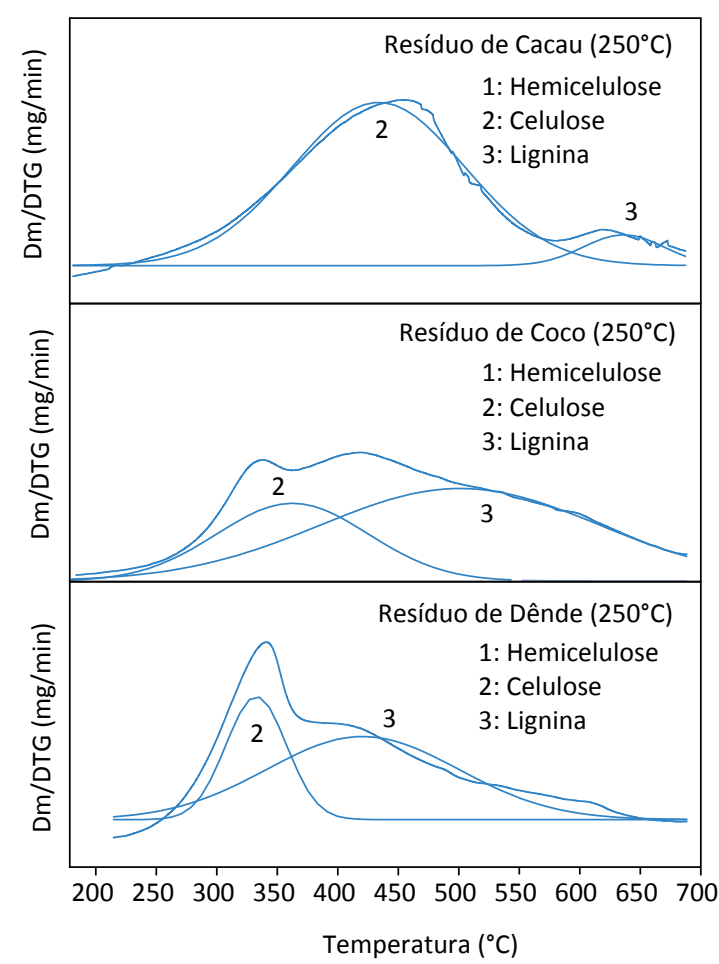

Dm/DTG: derivada da massa em função da temperatura que responde linearmente ao tempo.

Figura 2 - Termogravimetria derivativa e deconvolução para os resíduos de biomassas: (A) in natura; (B) torrificada a $230^{\circ} \mathrm{C}$; (C) torrificada a $240^{\circ} \mathrm{C}$; (D) torrificada a $250^{\circ} \mathrm{C}$. 
refação, o que justifica a diminuição da umidade e do teor de voláteis, com consequente aumento do teor de carbono fixo e do poder calorífico superior das amostras após a torrefação (ROWELL, 2005; CHEN et al., 2015; PELANDA et al., 2015; WANG et al., 2017a).

De acordo com as curvas termogravimétricas e de termogravimetria derivativa apresentadas na Figura 2, têm-se estimativas teóricas acerca da decomposição dos principais macrocomponentes das biomassas estudadas. A Figura 2A representa: a degradação da hemicelulose (curva 1); a da celulose (a curva 2); e a da lignina (curva 3). De acordo com as curvas de deconvolução, as biomassas in natura apresentam os picos correspondentes aos três macrocomponentes; porém, após sua torrefação a $230^{\circ} \mathrm{C}$, a presença do pico correspondente à hemicelulose passa a ser notada apenas no resíduo de cacau, não sendo mais visto nos resíduos de coco e de dendê. Para as duas outras temperaturas de torrefação, 240 e $250^{\circ} \mathrm{C}$, também não se observa a presença da hemicelulose, de acordo as curvas termogravimétricas.

As hemiceluloses são polissacarídeos que contêm uma grande quantidade de grupos hidroxila e, em razão da presença de ligações de hidrogênio nesses grupos, quanto mais hemicelulose houver na composição da biomassa, maior será a absorção de água e menor será o poder calorífico superior da mesma. A estimativa dos produtos de desvolatilização por meio das curvas de termogravimetria derivativa (DTG) (Tabela 2) também aponta, como esperado, para a diminuição do percentual de hemicelulose nas biomassas após a torrefação, evidenciando mais uma vez que o tratamento térmico contribui realmente para o aumento do PCS dos resíduos estudados.

Nas curvas de DTG para as biomassas de cacau e de dendê in natura aparecem picos que podem estar relacionados a microcomponentes, como óleos e ceras, presentes nos resíduos analisados (CONAB, 2006).

Na Tabela 2 são apresentados os dados referentes à desvolatilização dos resíduos de biomassa.

Comparando-se os resultados na Figura 2 com a Tabela 2, observa-se que, quando as curvas referentes à hemicelulose diminuem, o teor de umidade decresce, elevando o poder calorífico das biomassas, com teores diminuindo de $24 \%$ para biomassa in natura a valores não detectáveis (<LD limite de detecção do equipamento) para as amostras torrificadas, como observado na Tabela 2 (CHAO et al., 2016).

Arias et al. (2008) utilizaram a análise termogravimétrica a fim de estudar a reatividade de amostras de biomassa, tendo encontrado também apenas dois picos na curva das biomassas torrificadas. Chen e Kuo (2010) mencionaram as vantagens da análise de materiais torrificados por termogravimetria em razão da grande precisão com que é possível medir o processo de perda de massa das biomassas, o que foi de grande relevância para o trabalho dos autores que investigaram, especialmente, 0 impacto dos processos de torrefação e pirólise nas estruturas lignocelulósicas de biomassas (STELTE et al., 2013; PRINS et al., 2006). Para os resíduos das biomassas torrificadas, observa-se, na Tabela 2, o aumento do teor de celulose e lignina em torno de 40 para $80 \%$ para

Tabela 2 - Produtos de desvolatilização dos resíduos de biomassa in natura, torrificados a 230,240 e $250{ }^{\circ} \mathrm{C}$.

\begin{tabular}{|c|c|c|c|c|c|}
\hline Biomassa & Reação (zonas) & Biomassa seca & $\begin{array}{c}\text { Torrificada } \\
230^{\circ} \mathrm{C}\end{array}$ & $\begin{array}{l}\text { Torrificada } \\
240^{\circ} \mathrm{C}\end{array}$ & $\begin{array}{l}\text { Torrificada } \\
250^{\circ} \mathrm{C}\end{array}$ \\
\hline Coco & \multirow{3}{*}{$\begin{array}{c}1 \\
\text { (Hemicelulose \%) }\end{array}$} & 3,9 & $<$ L.D*. & $<$ L.D*. & $<$ L.D*. \\
\hline Cacau & & 24,4 & 12,4 & < L.D*. & $<$ L.D*. \\
\hline Dendê & & 5,8 & $<$ L.D*. & $<$ L.D*. & $<$ L.D*. \\
\hline Coco & \multirow{3}{*}{$\begin{array}{c}2 \\
\text { (Celulose \%) }\end{array}$} & 38,0 & 34,2 & 47,8 & 35,1 \\
\hline Cacau & & 44,4 & 57,6 & 19,3 & 68,2 \\
\hline Dendê & & 56,0 & 19,8 & 81,0 & 75,9 \\
\hline Coco & \multirow{3}{*}{$\begin{array}{c}3 \\
\text { (Lignina \%) }\end{array}$} & 57,9 & 65,7 & 52,1 & 64,8 \\
\hline Cacau & & 57,2 & 30,0 & 80,7 & 31,7 \\
\hline Dendê & & 38,2 & 80,2 & 18,9 & 24,1 \\
\hline
\end{tabular}

* Limite de detecção do equipamento de análise térmica é <0,0001 mg. 
as duas biomassas, sendo tal fato atribuído à diminuição dos teores de hemicelulose, corroborando com dados obtidos por Wang et al. (2017a).

As biomassas também foram caracterizadas por meio de MEV, de modo a estudar sua morfologia e modificações cau- sadas pelo tratamento térmico em suas superfícies. As micrografias foram feitas com aumento de cerca de 500 vezes.

Na Figura 3A, micrografias para a biomassa de coco, é possível notar que a superfície da biomassa in natura é consideravelmente menos rugosa, quando comparada com

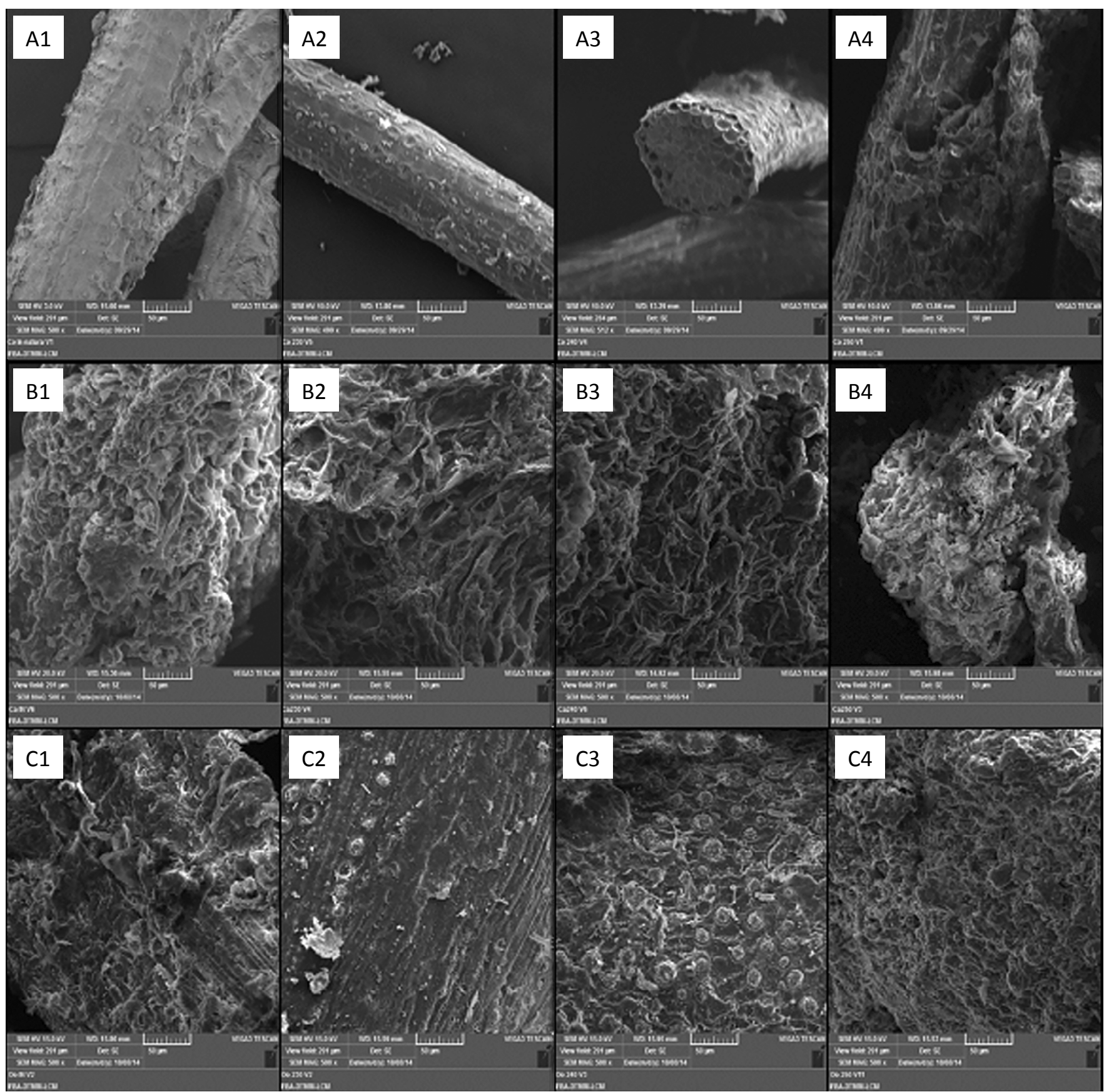

Figura 3 - Microscopia eletrônica de varredura para a biomassa de coco: A1, seca; de A2 a A4 torrificada a 230, 240 e $250^{\circ} \mathrm{C}$, respectivamente. Para a biomassa de cacau: B1, seca; de B2 a B4, torrificada a 230,240 e $250^{\circ} \mathrm{C}$, respectivamente. Para a biomassa de dendê: $\mathrm{C} 1$, seca; de $\mathrm{C} 2$ a C4, torrificada a 230,240 e $250^{\circ} \mathrm{C}$, respectivamente. 
as dos resíduos torrificados. As biomassas torrificadas tendem a possuir uma superfície fragilizada e descamada em razão do tratamento térmico que torna o material mais rígido. Como já foi dito, a hemicelulose é degradada durante a torrefação; sendo ela o principal componente responsável pela flexibilidade do vegetal, ao ser degradada, ocorre a perda dessa característica. A desvolatilização da hemicelulose após a torrefação também é evidenciada pelo desaparecimento dos pontos brancos observados no MEV da biomassa torrificada a $230^{\circ} \mathrm{C}$. Na biomassa torrificada a 240 e a $250^{\circ} \mathrm{C}$, pode-se observar sulcos, nos quais a hemicelulose se encontrava antes de sua degradação térmica pela torrefação (MISLENOVIC et al., 2015).

As micrografias para a biomassa de cacau (Figura 3B) não demonstraram haver muita diferença entre as su- perfícies das fibras in natura e das fibras torrificadas, embora haja diferenças nas propriedades das amostras analisadas (in natura e torrificadas), uma vez que já foram mencionadas as diferenças entre os teores de umidade, de voláteis, de cinzas, de carbono fixo e o PCS delas.

Para a biomassa de dendê, as micrografias (Figura 3C) apresentaram certas mudanças dos resíduos in natura para os resíduos torrificados. Essas mudanças, no entanto, não foram tão perceptíveis quanto para a biomassa de coco, mas é possível notar a presença de pequenos pontos esbranquiçados, possivelmente hemicelulose, nas micrografias da biomassa in natura e torrificada a 230 e a $240^{\circ} \mathrm{C}$, mas não estão presentes na biomassa torrificada a $250^{\circ} \mathrm{C}$.

\section{CONCLUSÕES}

De acordo com os resultados, pode-se verificar a influência do condicionamento térmico por meio da torrefação nas propriedades energéticas das biomassas de coco, cacau e dendê, bem como a comparação desses resultados com os obtidos para as biomassas in natura. Os dados obtidos permitem afirmar que o processo de torrefação melhorou o potencial energético dos resíduos estudados, causando o aumento de seu poder calorífico superior.

Das biomassas analisadas, a de coco foi a que apresentou o maior PCS em todas as temperaturas de torrefação e também in natura. A melhor temperatura de torrefação para as biomassas de coco e de dendê foi $250^{\circ} \mathrm{C}$; para a biomassa de cacau, foi $240^{\circ} \mathrm{C}$. Para a biomassa de coco torrificada, o aumento médio no
PCS, com relação à biomassa in natura, foi de $24,1 \%$; para a de cacau, $21,3 \%$; e para a de dendê, $35,2 \%$.

Com base nesses dados, pode-se afirmar que o processo de condicionamento da biomassa, por meio do processo de torrefação, melhora consideravelmente o poder energético dos resíduos de coco, de cacau e de dendê, por meio da degradação de componentes que os fazem adsorver umidade e, consequentemente, abaixam seu PCS. A comparação dos valores de PCS experimentais com alguns encontrados na literatura permite concluir que as biomassas estudadas podem ser aproveitadas como biocombustível sólido, uma vez que seus PCS são bastante próximos, e algumas vezes superiores, aos PCS de biomassas que já são utilizadas para a produção de carvão, o que torna o biocombustível sólido eficiente e sustentável do ponto de vista energético.

\section{REFERÊNCIAS}

A LAVOURA. Coco: Qual destino dar à casca? A Lavoura, n. 690, p. 26-30, 2012.

AGAR, D.; GIL, J.; SANCHEZ, D.; ECHEVERRIA, I.; WIHERSAARI, M. Torrefied versus conventional pellet production A comparative study on energy and emission balance based on pilot-plant data and EU sustainability criteria. Applied Energy, n. 138, p. 621-630, 2015.

ALMEIDA, G.; BRITO, J. O.; PERRE, P. Alterations in energy properties of eucalyptus wood and bark subjected to torrefaction: The potential of mass loss as a synthetic indicator. Bioresource Technology, n. 101, p. 9778-9784, 2010.

AMERICAN SOCIETY FOR TESTING AND MATERIALS (ASTM). ASTM D 1102-84: Standard Test Method for Ash in Wood. United States: ASTM International, 1995. 
AMERICAN SOCIETY FOR TESTING AND MATERIALS (ASTM). ASTM E 871-82: Standard Test Method for Moisture Analysis of Particulate Wood Fuels. United States: ASTM International, 1998a.

. ASTM E 872-82: Standard Test Method for Moisture Analysis of Particulate Wood Fuels. United States: ASTM International, 1998b.

ARIAS, B.; PEVIDA, C.; FERMOSO, J.; PLAZA, M. G.; RUBIERA, F.; PIS, J. J. Influence of torrefaction on the grindabilty and reactivity of wood biomass. Fuel Processing Technology, n. 89, p. 169-175, 2008.

AZADI, P.; MALINA, R.; BARRETT, S. R. H.; KRAFT, M. The evolution of the biofuel science. Renewable and sustainable energy reviews, v. 76, p. 1479-1484, 2017.

BERGMAN, P. C. A.; KIEL, J. H. A. Torrefaction for biomass upgrading. 14th European Biomass Conference e Exhibition, p. 17-21, 2005.

BRASIL. Ministério de Minas e Energia. Plano Nacional de Energia 2030. Brasília: Ministério de Minas e Energia/Empresa de Pesquisa Energética, 2007.

BROSTROM, M.; NORDIN, A.; POMMER, L.; BRANCA, C.; BLASI, D. Influence of torrefaction on the devolatilization and oxidation kinetics of wood. Journal of Analytical and Applied Pyrolysis, n. 96, p. 100-109, 2012.

BURATTI, C.; BARBANERA, M.; LASCARO, E.; COTANA, F. Optimization of torrefaction conditions of coffee industry residues using desirability function approach. Waste Management, p. 1-12, 2017. Disponível em: <http://dx.doi. org/10.1016/j.wasman.2017.04.012>. Acesso em: 02 jun. 2017.

CHAI, L.; SAFFRON, C. M. Comparing pelletization and torrefaction depots: Optimization of depot capacity and biomass moisture to determine the minimum production cost. Applied Energy, n. 163, p. 387-395, 2016.

CHAO, L.; YUAN, X.; JIANG, L.; LI, C.; XIAO, Z.; HUANG, Z.; CHEN, X.; ZENG, G.; LI, H. Thermogravimetric characteristics and kinetics analysis of oil cake and torrefied biomass blends. Fuel, v. 175, p. 129-136, 2016.

CHAO, L.; YUAN, X.; LI, H.; LI, C.; XIAO, Z.; JIANG, L.; HUANG, B.; XIAO, Z.; CHEN, X.; WANG, H.; ZENG, G. Complementary effects of torrefaction and co-pelletization: Energy consumption and characteristics of pellets. Bioresource Technology, v. 185, p. 254-262, 2015.

CHEN, H.; CHEN, X.; QUN, Y.; WEI, J.; LIU. Effect of torrefaction on the properties of rice straw high temperature pyrolysis char: Pore structure, aromaticity and gasification activity. Bioresource Technology, v. 228, p. 241-249, 2017.

CHEN, W.; PENG, J.; BI, X. T. A state-of-the-art review of biomass torrefaction, densification and applications. Renewable and Sustainable Energy Reviews, n. 44, p. 847-866, 2015.

CHEN, W. H.; KUO, P. C. A study on torrefaction of various biomass materials and its impact on lignocellulosic structure simulated by a thermogravimetry. Energy, n. 35, p. 2580-2586, 2010.

CHEN, Y.; CAO, W.; ATREYA, A. An experimental study to investigate the effect of torrefaction temperature and time in pyrolysis of centimenter-scale pine wood particles. Fuel Processing Technology, v. 153, p. 74-80, 2016.

CIOLKOSZ, D.; WALLACE, R. A review of torrefaction for bioenergy feedstock production. Biofuels Bioproduction Biorefineries, v. 5, n. 3, p. 317-29, 2011.

COMISSÃO EXECUTIVA DO PLANO DA LAVOURA CACAUEIRA (CEPLAC). Cacau: História e Evolução. Disponível em: <http://www.ceplac.gov.br/radar/radar_cacau.htm>. Acesso em: 30 abr. 2014.

COMPANHIA NACIONAL DE ABASTECIMENTO (CONAB). Dendeicultura da Bahia.Bahia: CONAB, 2006. 
CONFEDERAÇÃO NACIONAL DA INDÚSTRIA (CNI). Matriz energética: Cenários, oportunidades e desafios. Brasília: CNI, 2007. 82 p. CORTEZ, L. A. B.; LORA, E. E. S.; GÓMEZ, E. O. Biomassa para energia. Campinas: Editora Unicamp, 2008.

DENG, J.; WANG, G.; KUANG, J.; ZHANG, Y.; LUO, Y. Pretreatment of agricultural residues for co-gasification via torrefaction. Journal of Analytical and Applied Pyrolysis, n. 86, p. 331-337,2009.

DODDAPANENI, T. R. K. C.; KONTTINEN J.; HUKKA, T. I.; MOILANEN, A. Influence of torrefaction pretreatment on the pyrolysis of Eucalyptus clone: A study on kinetics, reaction mechanism and heat flow. Industrial Cropsand Products, n. 92, p. 244-254,2016.

EMPRESA BRASILEIRA DE PESQUISA AGROPECUÁRIA (EMBRAPA). Embrapa planeja aplicações para resíduos de dendê. Brasil: Embrapa, 2012. Disponível em: <http://www.canalrural.com.br/noticias/agricultura/embrapa-planejaaplicacoes-para-residuos-dende-38764>. Acesso em: 30 abr. 2014.

IBRAHIM, R. H. H.; DARVELL, L. I.; JONES, J. M.; WILLIAMS, A. Physicochemical characterization of torrefied biomass. Journal of Analytical and Applied Pyrolysis, n. 103, p. 21-30, 2013.

LASEK, J. A.; KOPCZYNSKI, M.; JANUSZ, M.; ILUK, A.; ZUWALA, J. Combustion properties of torrefied biomass obtained from flue gas-enhanced reactor. Energy, v. 119, p. 362-368, 2017.

LOBÃO, E. A crise do petróleo e os biocombustíveis. 2008. Disponível em: <http://www1.folha.uol.com.br/fsp/opiniao/ fz2707200809.htm>. Acesso em: 28 out. 2014.

MISLJENOVIC, N.; MOSBYE, J.; SCHÜLLER, R. B.; LEKANG, O.-I.; SALAS-BRINGAS, C. Physical quality and surface hydration properties of wood based pellets blended with waste vegetable oil. Fuel Processing Technology, n. 134, p. 214-222, 2015.

MORORÓ, R. C. Aproveitamento de derivados, subprodutos e resíduos do cacau. In: VALLE, R. R. (Ed.). Ciência, tecnologia e manejo do cacaueiro. Ilhéus: Ceplac, p. 371- 421, $2007 .$.

MUIZNIECE, I.; KLAVINA, K.; BLUMBERGA, D. The impact of torrefaction on coniferous forest residue fuel. Energy Procedia, n. 95, p. 319-323, 2016.

NAKASHIMA, G. T.; MARTINS, M. P.; SILVA, D. A.; CHRISOSTOMO, W.; YAMAJI, F. M. Aproveitamento de resíduos vegetais para a produção de briquetes. Revista Brasileira de Ciências Ambientais, n. 34, p. 22-29, 2014.

NAM, S. B.; PARK, Y. S.; KIM, D. J.; GU, J. H. Torrefaction reaction characteristic of various biomass waste on pilot scale of torrefaction reaction system. Procedia Environmental Sciences, n. 35, p. 890-894, 2016.

NANDA, S.; ISEN, J.; DALAI, A. K.; KOZINSKI, J. A. Gasification of fruit and agro-food residues in supercritical water. Energy Conversion and Management, v. 110, p. 296-306, 2016.

PARIKH, J.; CHANNIWALA, S. A.; GHOSAL, G. K. A correlation for calculating HHV from proximate analysis of solid fuels. Fuel, n. 84, p. 487-494, 2005.

PELANDA, K. A.; POTULSKI, D. C.; SILVA, D. A.; FERRAZ, F. A. Avaliação das possíveis implicações do uso de diferentes biomassas florestais como biocombustivel em geradores de vapor. Ciência da Madeira (Brazilian Journal of Wood Science), v. 6, n. 2, p. 112-121, 2015. DOI: 10.12953/2177-6830/rcm.v6n2p112-121

PRINS, M. J.; PTASINSKI, K. J.; JANSSEN, F. J. J. G. More efficient biomass gasification via torrefaction. Energy, n. 31, p. 3458-3470, 2006.

PROSKURINA, S.; HEINIMÖ, J.; SCHIPFER, F.; VAKKILAINEN, E. Biomass for industrial applications: The role of torrefaction. Renewable Energy, n. 111, p. 265-274, 2017. 
RAMOS, C.; VENTURA, J. E. A energia solar em Portugal: Potencialidades e diferenciação regional. In: CONGRESSO DA GEOGRAFIA PORTUGUESA, 3., 1997, Porto. Artigo... Porto: Edições Colibri e Associação Portuguesa de Geógrafos, 1999. p. 453-461.

ROWELL, R. M. Handbook of Wood Chemistry and Wood Composites. Flórida: CRC, 2005.

RUDOLFSSON, M.; BORÉN, E.; POMMER, L.; NORDIN, A.; LESTANDER, T. Combined effects of torrefaction and pelletization parameters on the quality of pellets produced from torrefied biomass. Applied Energy, v. 191, p. 414-424, 2017.

SABIL, K. M.; AZIZ, M. A.; LAL, B.; UEMURA, Y. Effects of torrefaction on the physiochemical properties of oil palm empty fruit bunches, mesocarp fiber and kernel shell. Biomass and Bioenergy, n. 56, p. 351-360, 2013.

SALES, I. Resíduos do coco têm uso sustentável. Diário do Nordeste, Gestão Ambiental, 2011. Disponível em: <http:// diariodonordeste.verdesmares.com.br/cadernos/negocios/residuos-do-coco-tem-uso-sustentavel-1.642525>. Acesso em: 30 abr. 2014.

SANTOS, R. C.; CARNEIRO, A. C. O.; VITAL, B. R.; CASTRO, R. V. O.; VIDAURRE, G. B.; TRUGUILHO, P. F.; CASTRO, A. F. N. M. Influência das propriedades químicas e da relação Siringil/Guaiacil da madeira de eucalipto na produção de carvão. Ciência Florestal, v. 26, n. 2, p. 657-669, 2016.

SILVA, A. G. Condicionamento de resíduo de Eucalyptusgrandis para produção de biocombustivel sólido. $131 \mathrm{f}$. Tese (Doutorado em Química) - Instituto de Química, Universidade Federal da Bahia, Salvador, 2013.

SILVA, C. R. A. da et al. A biomassa como alternativa energética para o Brasil. Revista Brasileira de Ciências Ambientais, n. 2, p. 25-36, 2005.

STELTE, W.; NIELSEN, N. P. K.; HANSEN, H. O.; DAHL, J.; SHANG, L.; SANADI, A. R. Pelletizing properties of torrefied wheat straw. Biomassa e Bioenergia, n. 53, p. 105-112, 2013.

SUPERINTENDÊNCIA DE ESTUDOS ECONÔMICOS E SOCIAIS DA BAHIA (SEI). Sistema de informações municipais: economia > agropecuária, silvicultura e extrativa vegetal > cultura por município > quantidade produzida. Disponível em: < http://sim.sei.ba.gov.br/sim/tabelas.wsp>. Acesso em: 17 fev. 2015.

SYAMSIRO, M.; SAPTOADI, H.; TAMBUNAN, B. H.; PAMBUDI, N. A. A preliminary study on use of cocoa pod husk as a renewable source of energy in Indonesia. Energy for Sustainable Development, n. 16, p. 74-77, 2012.

TAVARES, S. R. L.; SANTOS, T. E. Uso de Diferentes Fontes de Biomassa Vegetal para Produção de Biocombustíveis Sólidos. Holos, p. 19-27, 2013.

VIEIRA, A. C. Caracterização da Biomassa Proveniente de Resíduos Agrícolas. 72 f. Dissertação (Mestrado em Energia na Agricultura) - Universidade Estadual do Oeste do Paraná, Cascavel, 2012.

WANG, L.; BARTA-RAJNAI, E.; SKREIBERG, O.; KHALIL, R.; CZÉGÉNY, Z.; JAKAB, E.; BARTA, Z.; GRONLI, M. Impact of torrefaction on woody biomass properties. Energy Procedia, n. 105, p. 1149-1154, 2017a.

WANG, S.; DAI, G.; RU, B.; ZHAO, Y.; WANG, X.; XIAO, G.; LUO, Z. Influence of torrefaction on the characteristics and pyrolysis behavior of cellulose. Energy, v. 120, p. 864-871, 2017b.

WANG, S.; DAI, G.; RU, B.; ZHAO, Y.; WANG, X.; ZHOU, J.; LUO, Z.; CEN, K. Effects of torrefaction on hemicellulose structural characteristics and pyrolysis behaviors. Bioresource Technology, v. 218, p. 1106-1114, 2016.

YUE, Y.; SINGH, H.; SINGH, B.; MANI, S. Torrefaction of sorghum biomass to improve fuel properties. Bioresource Technology, v. 232, p. 372-379, 2017. 